\section{Reversible cerebral vasoconstriction syndrome promptly diagnosed with magnetic resonance imaging including magnetic resonance angiography during immunosuppressive therapy in a 16-year-old girl with refractory cytopenia of childhood}

Hideaki Ueki, ${ }^{1}$ Yasushi Sanayama, ${ }^{2}$ Akiyo Miyajima, ${ }^{1}$ Taichiro Tsuchimochi, Shunji Igarashi, ${ }^{1}$ Shosuke Sunami ${ }^{1}$

'Department of Pediatric Hematology and Oncology, ${ }^{2}$ Department of Radiology, Japanese Red Cross Narita Hospital, Narita, Chiba, Japan

\section{Abstract}

Reversible cerebral vasoconstriction syndrome (RCVS) is a syndrome characterized by severe headache with segmental vasoconstriction of the cerebral arteries that resolves within 12 weeks. A 16-year-old girl with refractory cytopenia of childhood, who was receiving the immunosuppressant cyclosporine, developed severe headache and was diagnosed with RCVS using magnetic resonance imaging, including magnetic resonance angiography (MRA). MRA is a non-invasive and very effective technique for diagnosing RCVS. MRA should be performed at the onset of severe headache during immunosuppressant administration for children with hematological disorders and may prevent sequelae such as posterior reversible encephalopathy syndrome or ischemic attack.

\section{Introduction}

Reversible cerebral vasoconstriction syndrome (RCVS) is a syndrome characterized by the sudden onset of severe headache, often called thunderclap headache, with multifocal segmental vasoconstriction of cerebral arteries, identifiable as a beaded appearance on imaging modalities. Reversibility of these angiographic abnormalities is seen within 12 weeks after onset under appropriate therapy, including cessation of causative medicines and administration of a calcium blocker such as lomerizine. ${ }^{1-7}$ The pathophysiology of RCVS remains uncertain, although alterations in cerebrovascular tone are thought to represent a key underlying mechanism. ${ }^{1}$ Many substances, including immunosuppressants, vasoactive substances, and blood products, are thought to cause onset of RCVS. ${ }^{8}$ Timely and accurate diagnosis of RCVS is required for appropriate care. Delays in diagnosis may cause deterioration of symptoms and concomitant excessive diagnostic tests. However, diagnosis can be difficult because of signs and symptoms overlapping with those of better known disorders of the central nervous system, such as posterior reversible encephalopathy syndrome (PRES). ${ }^{3,4,9-13}$ In patients with hematological disease, calcineurin inhibitors such as cyclosporine and tacrolimus have been used in immunosuppressive therapy (IST) for the treatment of aplastic anemia, and the related disorder, refractory cytopenia of childhood (RCC), and in prophylactic treatment for graft-versus-host disease after hematopoietic cell transplantation. As one of the disorders leading to headache during the use of calcineurin inhibitors, PRES has often been reported. Although PRES is sometimes complicated by $\mathrm{RCVS},{ }^{13}$ very few reports have described RCVS in patients under 18 years old with hematological disease. We report the case of a 16-year-old girl with RCVS that developed during immunosuppressive therapy for RCC and was promptly diagnosed using magnetic resonance angiography (MRA), a group of techniques based on magnetic resonance imaging (MRI), and successfully treated.

\section{Case Report}

A 13-year-old girl visited our hospital with a 3-day history of fever and abdominal pain. Blood examinations revealed pancytopenia, and she was admitted to our hospital. Bone marrow findings showed hypoplasia and dysplasia of more than $10 \%$ of myeloid lineage. Gbanding of marrow cells revealed a 46,XX karyotype in all of the 10 cells analyzable, and fluorescence in situ hybridization detected neither monosomy 7 nor trisomy 8 . Based on these findings, RCC (a subtype of myelodysplastic syndrome) was diagnosed. Because cytopenia was not severe, she was observed in our outpatient clinic without treatment. After observation for 41 months, at 16 years old, the cytopenia worsened to the point of dependency on blood transfusion and the patient was admitted to our hospital for IST. From day 2 of hospitalization, IST was performed using rabbit antithymocyte globulin (Thymoglobulin; Sanofi K.K., Tokyo, Japan) at $3.5 \mathrm{mg} / \mathrm{kg} /$ day (for 5 days), cyclosporine A (CsA) started at 6 $\mathrm{mg} / \mathrm{kg} /$ day and adjusted to the range of 100-200 $\mathrm{ng} / \mathrm{mL}$ (for 180 days), and methylprednisolone at $2 \mathrm{mg} / \mathrm{kg}$ (full dose for 7 days, tapered down for 3 weeks, then suspended). On day 13 of
Correspondence: Hideaki Ueki, Department of Pediatric Hematology and Oncology, Japanese Red Cross Narita Hospital, 90-1, Iida-cho, Narita, Chiba 286-8523, Japan

Tel.: +81.476.22.2311 - Fax: +81.476.22.6477.

E-mail: uekih0209@gmail.com

Key words: Reversible cerebral vasoconstriction syndrome; Immunosuppressive therapy; Magnetic resonance angiography; Refractory cytopenia of childhood; Children.

Acknowledgements: we wish to thank the staff of our institution who participated in the treatment of this patient.

Contributions: $\mathrm{HU}$ treated the patient and wrote the manuscript; YS contributed diagnosing the patient radiologically; AM, TT and SI treated the patient as members of clinical team; SS considerably advised me on writing this manuscript.

Conflict of interest: the authors declare no potential conflict of interest.

Received for publication: 29 June 2016. Accepted for publication: 11 October 2016.

This work is licensed under a Creative Commons Attribution-NonCommercial 4.0 International License (CC BY-NC 4.0).

(C) Copyright H. Ueki et al., 2016

Licensee PAGEPress, Italy

Hematology Reports 2016; 8:6673

doi:10.4081/hr.2016.6673

hospitalization, throbbing headache arose in the left frontal to posterior region of the head. This headache was not severe at onset but gradually worsened over 4 days to $7 / 10$ on the numeric rating scale, leading to sleep disturbance. 0n day 17 of hospitalization, MRI including MRA was performed. MRA detected segmental constriction as the beaded appearance of bilateral internal carotid arteries and bilateral anterior cerebral arteries (Figure 1). T1- and T2-weighted imaging, fluid-attenuated inversion recovery imaging, and diffusionweighted imaging as other MRI techniques revealed no abnormalities of the brain. On day 18 of hospitalization, CsA was suspended and administration of the calcium blocker lomerizine was started at $10 \mathrm{mg} /$ day. Headache promptly improved, resolving by day 23 of hospitalization (5 days after starting lomerizine administration). MRA on day 26 of hospitalization showed regression of vasoconstriction (Figure 2). CsA was restarted on day 57 of hospitalization (day 44 after onset of headache) with continuation of lomerizine, because of the need for this agent in the treatment of RCC. No recurrence of headache has been seen for one year after discharge. 


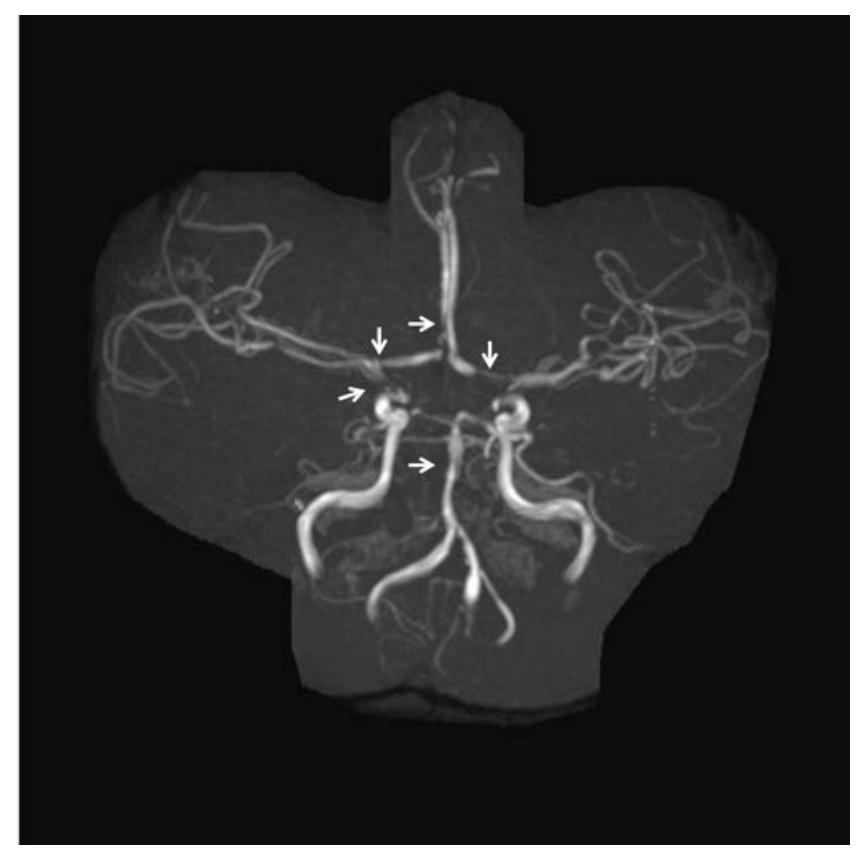

Figure 1. Magnetic resonance angiography (MRA) on day 17 of hospitalization (4 days after onset of headache). MRA shows segmental constriction (beaded appearance) of bilateral internal carotid arteries and bilateral anterior cerebral arteries (arrows).

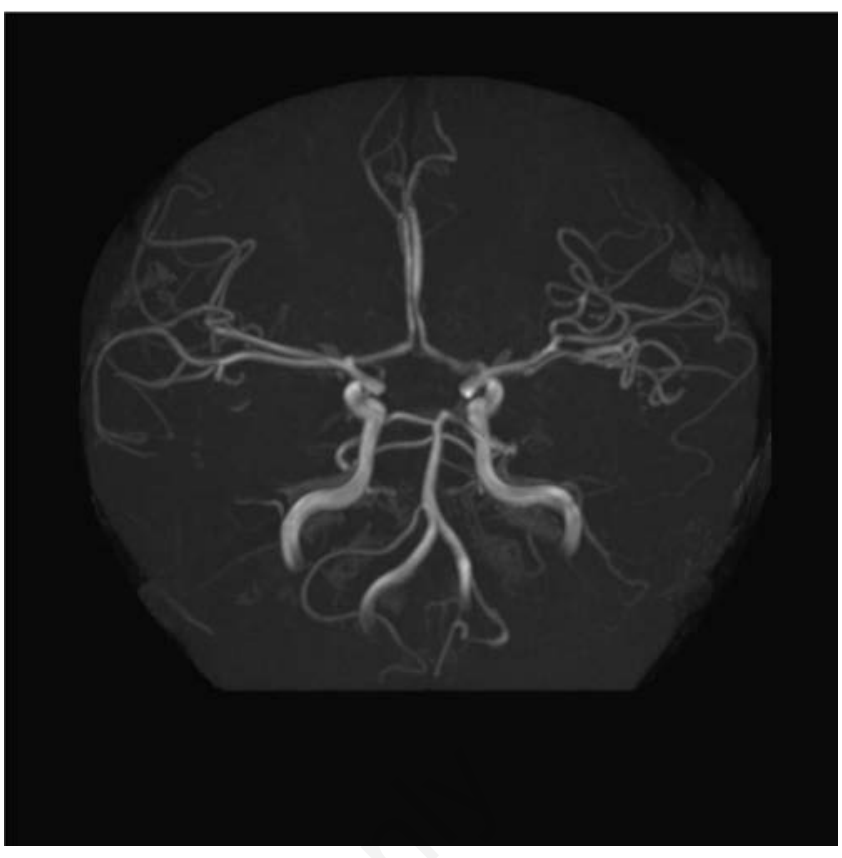

Figure 2. Magnetic resonance angiography (MRA) on day 26 of hospitalization (13 days after onset of headache). MRA shows regression of vasoconstriction.

\section{Discussion}

We promptly diagnosed and successfully treated RCVS during IST in this 16-year-old girl with RCC using MRI, including MRA. Imaging modalities used in the management of RCVS include transcranial Doppler ultrasonography, non-contrast computed tomography (CT), CT angiography, MRA, vessel wall imaging, and catheter angiography. ${ }^{2}$ Cerebral angiography is the standard criterion for the detection of cerebral vasoconstriction, but noninvasive techniques such as MRA are increasingly being used in clinical practice. MRA is an effective technique for diagnosing and monitoring the evolution of RCVS-related vasoconstriction. ${ }^{14}$ We used MRI including MRA for screening our patient with severe headache, and MRA revealed segmental vasoconstriction as a beaded appearance of the left internal carotid artery, leading to a diagnosis of RCVS, but T1- and T2-weighted, fluid-attenuated inversion recovery imaging, and diffusionweighted imaging revealed no abnormalities. MRA is in relatively widespread use and is a non-invasive modality, which is useful in screening for headache. However, when PRES is suspected as a cause of headache, MRA is not generally performed for screening, because PRES can be diagnosed by MRI sequences without MRA. As a result, some cases of RCVS may be misdiagnosed as PRES not complicated with RCVS.

RCVS is closely related to PRES and ischemic stroke. Ducros and colleagues reported that $9-38 \%$ of patients with RCVS had PRESlike lesions. ${ }^{15}$ Chen and colleagues found that vasoconstrictions are important determinants for PRES and ischemic stroke. ${ }^{14}$ Timely diagnosis and appropriate treatment for RCVS may prevent the onset of these clinical problems.

Reports of RCVS in children with hematological disease are very rare. A PubMed search for cases of RCVS in children with hematological diseases, using the search terms reversible cerebral vasoconstriction syndrome [Title/Abstract], and children [Title/Abstract] or pediatric [Title/Abstract], yielded only one report of RCVS. ${ }^{16}$ However, some cases of RCVS may go unrecognized, considering the relationship between RCVS and PRES. Surveys of cases from multiple centers are needed to clarify the actual incidence of RCVS in children with hematological disease.

\section{Conclusions}

MRA should be included in diagnostic modalities for severe headache during administration of causative medicines such as calcineurin inhibitors, for the purpose of prompt diagnosis and treatment of RCVS.

\section{References}

1. Miller TR, Shivashankar R, Mossa-Basha M, Gandhi D. Reversible cerebral vasoconstriction syndrome, part 1: epidemiology, pathogenesis, and clinical course. AJNR Am J Neuroradiol 2015;36:1392-9.

2. Miller TR, Shivashankar R, Mossa-Basha M, Gandhi D. Reversible cerebral vasoconstriction syndrome, part 2: diagnostic work-up, imaging evaluation, and differential diagnosis. AJNR Am J Neuroradiol 2015;36:1580-8.

3. Ducros A. L37. Reversible cerebral vasoconstriction syndrome: distinction from CNS vasculitis. Presse Med 2013;42:602-4.

4. Ducros A, Bousser MG. Reversible cerebral vasoconstriction syndrome. Pract Neurol 2009;9:256-67.

5. Gupta S, Zivadinov R, Ramasamy D, Ambrus JL Jr. Reversible cerebral vasoconstriction syndrome (RCVS) in antiphospholipid antibody syndrome (APLA): the role of centrally acting vasodilators. Case series and review of literature. Clin Rheumatol 2014;33:1829-33.

6. Marder CP, Donohue MM, Weinstein JR, Fink KR. Multimodal imaging of reversible cerebral vasoconstriction syndrome: a series of 6 cases. Am J Neuroradiol 2012;33:1403-10.

7. Sheikh HU, Mathew PG. Reversible cere- 
bral vasoconstriction syndrome: updates and new perspectives. Curr Pain Headache Rep 2014;18:414.

8. Ducros A, Bousser MG. Reversible cerebral vasoconstriction syndrome. Pract Neurol 2009;9:256-67.

9. Calabrese LH, Dodick DW, Schwedt TJ, Singhal AB. Narrative review: reversible cerebral vasoconstriction syndromes. Ann Intern Med 2007;146:34-4.

10. Calic Z, Choong H, Schlaphoff G, CappelenSmith C. Reversible cerebral vasoconstriction syndrome following indomethacin.
Cephalalgia 2014;34:1181-6.

11. Edlow BL, Kasner SE, Levine JM, et al. Reversible cerebral vasoconstriction syndrome associated with subarachnoid hemorrhage. Neurocrit Care 2007;7:203-10.

12. Grooters GS, Sluzewski M, Tijssen CC. How often is thunderclap headache caused by the reversible cerebralvasoconstriction syndrome? Headache 2014;54:732-5.

13. Akazawa $\mathrm{Y}$, Inaba $\mathrm{Y}$, Koike $\mathrm{K}$, et al. Reversible cerebral vasoconstriction syndrome and posterior reversible encephalopathy syndrome in a boy with
Loeys-Dietz syndrome. Am J Med Genet A 2015;167A:2435-9.

14. Chen SP, Fuh JL, Wu JC, et al. Magnetic resonance angiography in reversible cerebral vasoconstriction syndromes. Ann Neurol 2010;67:648-56.

15. Ducros A. Reversible cerebral vasoconstriction syndrome. Lancet Neurol 2012; 11:906-17.

16. Kazato Y, Fujii K, Oba H, et al. Reversible cerebral vasoconstriction syndrome associated with brain parenchymal hemorrhage. Brain Dev 2012;34:696-9. 